\title{
Histologie testiculaire et études méiotiques dans les stérilités de type non obstructif
}

\author{
Rahma GHALAMOUN-SLAIMI, Marie-Roberte GUICHAOUA
}

Laboratoire de Biologie de la Reproduction, Hôpital de la Conception, Marseille

\section{RESUME}

L'avènement de I'ICSI couplée à la biopsie testiculaire et le développement de nouvelles méthodes d'investigation de la spermatogenèse apportent un regain d'intérêt pour l'analyse de la spermatogenèse à partir de la biopsie testiculaire. Si on veut progresser dans le diagnostic étiologique, la connaissance des mécanismes et les risques pour l'embryon des azoospermies et des oligospermies sévères de type non obstructif, il est nécessaire d'établir le "phénotype testiculaire " de chaque patient. Pour cela, nous réalisons systématiquement chez tout patient qui a une biopsie testiculaire associée à une ICSI, une étude histopathologique, faite sur un fragment testiculaire de la taille d'un grain de riz prélevé au cours de la biopsie, et une étude méiotique réalisée sur le matériel non utilisé pour I'ICSI et contenant toutes les cellules germinales immatures.

L'étude histopathologique doit être à la fois quantitative et qualitative. Elle permet de classer les anomalies du testicule en 4 grands groupes de lésions : le syndrome des cellules de Sertoli seules, la hyalinisation des tubules, les lésions diffuses de la spermatogenèse, l'atrophie mixte du testicule. L'étude méiotique visualise les chromosomes dont le comportement reflète l'ètat d'altération de la spermatogenèse. Le marquage des complexes synaptonémaux avec l'anticorps COR1/SCP3 nous a permis de décrire plusieurs types d'anomalies que l'on observe avec des fréquences variables dans les spermatogenèses altérées, cependant des profils particuliers émergent dans les syndromes d'étiologie connue. Nous nous sommes particulièrement intéressés aux défauts d'appariement des chromosomes (asynapsis) au cours du stade pachytène de la prophase I de la méiose. Nous avons montré que les taux d'asynapsis étaient significativement plus élevés dans les azoospermies de type non obstructives (NO) que dans les obstructives ( $O$ ). Chez deux patients du groupe
NO qui avaient des taux particulièrement élevés, nous avons suggéré la présence d'un déficit primaire de la méiose. L'un de ces patients est porteur d'une microdélétion AZFc. Un taux élevé d'asynapsis a aussi été mis en évidence chez un patient porteur d'une microdélétion AZFb. Par ailleurs, quelque soit l'étiologie de la défaillance de la spermatogenèse, un arrêt partiel ou total de la méiose se produit au milieu du stade pachytène. A partir de ces travaux, nous avons proposé l'hypothèse que le point de contrôle du pachytène était situé au milieu de ce stade.

Mots clés : azoospermie, oligospermie, histologie testiculaire, méiose, asynapsis

\section{INTRODUCTION}

Depuis plus de 60 ans, un grand nombre d'interrogations et de prises de positions contradictoires ont émaillé l'histoire de la biopsie testiculaire. Cet examen, longtemps réalisé dans un double but, diagnostique et pronostique, à partir de l'étude histopathologique de la spermatogenèse, apportait peu de renseignements comparés aux risques de l'intervention chirurgicale et avait ainsi disparu de la liste des investigations de l'infertilité masculine. L'avènement de l'ICSI, couplée à la biopsie testiculaire, et le

\section{Correspondance :}

Pr Marie-Roberte GUICHAOUA - Laboratoire de Biologie de la Reproduction, Hôpital de la Conception, 147 Boulevard Baille, 13385 Marseille Cedex 05 - Email mguichaoua@ap-hm.fr 
développement de nouvelles méthodes d'analyse des cellules germinales telles que l'immunocytochimie et l'hybridation in situ, ont apporté un regain d'intérêt pour l'analyse de la spermatogenèse à partir de la biopsie testiculaire. Ainsi, l'indication de la biopsie testiculaire, réalisée dans un but thérapeutique, ne se pose plus actuellement.

C'est donc dans le cadre de ce geste thérapeutique que le matériel testiculaire peut être utilisé dans un but diagnostique et de recherche, le consentement éclairé du patient étant indispensable pour la réalisation de ces travaux. En effet, si l'on veut avancer dans le diagnostic étiologique, la connaissance des mécanismes et les risques pour l'embryon des azoospermies et des oligospermies sévères de type non obstructif, il est nécessaire de faire un état des lieux le plus précis et le plus détaillé possible de la spermatogenèse de ces patients, il faut établir le " phénotype testiculaire » de chaque patient. Deux techniques d'analyse de la spermatogenèse sont systématiquement réalisées dans notre laboratoire lorsqu'un patient a une biopsie testiculaire associée à une ICSI : l'étude histopathologique et l'étude méiotique. Ces deux études sont étroitement complémentaires l'une de l'autre et elles ont deux objectifs : 1) apprécier l'état de la spermatogenèse, et 2) tenter de trouver une cause ou orienter le diagnostic étiologique de cette infertilité.

\section{RAPPEL DE LA SPERMATOGENESE}

La Valette Saint George en 1876 [7] définissait la spermatogenèse comme le processus par lequel une spermatogonie souche donne naissance à un certain nombre de spermatozoïdes et qui peut être divisé en 3 phases, chacune ayant une fonction bien définie. 1) une phase de multiplication et de renouvellement des spermatogonies ; 2) une phase de méiose qui se déroule dans les spermatocytes I et II et qui s'achève par la formation de cellules haploïdes, les spermatides ; 3) une phase de maturation, la spermiogenèse au cours de laquelle les spermatides subissent une série de transformations nécessaires à la fécondation et qui donnent naissance aux spermatozoïdes. Les 1 ère et 3ème phases présentent des caractéristiques spécifiques de l'espèce, tandis que les spermatocytes au cours de la méiose ont des caractères morphologiques semblables dans de nombreuses espèces de mammifères et dans des espèces aussi éloignées de la nôtre que la levure Saccharomyces cerevisiae.

Les différents types cellulaires qui caractérisent la spermatogenèse constituent dans les tubes séminifères des associations bien définies qui forment le « cycle de l'épithélium séminifère " dans lequel une séquence cellulaire est répétée à l'infini [1]. Le cycle de l'épithélium séminifère est un phénomène histologique dynamique qui prend place avec le temps en un endroit donné de l'épithélium séminifère. Chez l'homme, le nombre de cellules germinales qui sont vues à la même étape de développement sur les coupes histologiques est relativement petit, de tels groupes cellulaires occupent des aires limitées de l'épithélium séminifère. L'évolution synchrone des cellules germinales au sein de ces groupes cellulaires résulte d'une part de la présence de ponts intercellulaires, d'autre part de facteurs sécrétés par les cellules de Sertoli, qui coordonnent l'évolution de 5 à 6 générations de cellules germinales. Ces notions rendent compte des variations cellulaires observées d'un tube à l'autre, chez les sujets normaux et chez les patients qui présentent des défaillances de la spermatogenèse et dont il faut tenir compte dans l'interprétation de l'histopathologie testiculaire.

\section{ETUDE HISTOPATHOLOGIQUE}

\section{Prélèvement et classification des lésions}

Lors du prélèvement chirurgical, il est indispensable qu'un fragment testiculaire soit immédiatement fixé et envoyé dans un laboratoire d'anatomopathologie. II est souhaitable d'avoir un échantillon par testicule, car dans $28 \%$ des cas, les lésions diffèrent d'un testicule à l'autre. L'existence d'atrophies mixtes du testicule nécessiterait le prélèvement de plusieurs fragments, mais ceci n'est pas envisageable en pratique, car il est indispensable de ne pas pénaliser le patient quant à la réalisation et aux résultats de l'ICSI. Dans cette même optique, la taille du ou des fragments prélevés ne doit pas dépasser la taille d'un grain de riz. La qualité de l'interprétation va donc dépendre du prélèvement, mais aussi de la fixation et de l'analyse des lames. Nous utilisons comme fixateur du formol à $4 \%$, tamponné par du PBS $1 \mathrm{X}$ et additionné de $25 \%$ d'éthanol pur.

La première analyse des lames est faites par les anatomopathologistes qui recherchent, en plus d'une anomalie de la spermatogenèse, des lésions précancéreuses. Puis, une lame de chaque testicule est envoyée au Laboratoire de Biologie de la Reproduction afin de faire une étude beaucoup plus fine des anomalies de la spermatogenèse, de la paroi des tubes séminifères et des cellules interstitielles. Une description détaillée qualitative et quantitative des caractéristiques testiculaires chez l'homme adulte normal, avec une évaluation du nombre moyen par tube séminifère de chaque catégorie cellulaire a été faite par Nistal et Paniaga [10]. L'analyse morphométrique assistée par ordinateur peut faciliter considérablement ces études à condition de posséder le matériel nécessaire pour sa réalisation 
[3]. Dans notre laboratoire, nous analysons environ 30 à 50 coupes de tubes séminifères par testicule, nous faisons une évaluation qualitative du diamètre moyen des tubes séminifères, de l'épaisseur de la lamina propria, des cellules péritubulaires et des amas de Leydig. Nous évaluons le nombre moyen par tube séminifère de spermatogonies, de spermatocytes $I$, de spermatides $\mathrm{Sa}+\mathrm{Sb}$ et de spermatides $\mathrm{Sc}+\mathrm{Sd}$.

Notre classification des lésions de la spermatogenèse se calque étroitement sur celle proposée par Nistal et Paniaga [10]. Quatre types de lésions sont fréquemment rencontrées : le syndrome SCO (Sertoli Cell Only), la hyalinisation tubulaire, les lésions diffuses de la spermatogenèse et les atrophies mixtes.

\section{a) Le syndrome SCO (Figure 1A)}

Il est retrouvé chez $13 \%$ des patients présentant une azoospermie. Il se manifeste par une absence totale de cellules germinales dans les tubes séminifères où seules les cellules de Sertoli sont présentes. Celles-ci peuvent être morphologiquement normales ou montrer des anomalies principalement nucléaires.

\section{b) La hyalinisation tubulaire (Figures 1B et $\mathrm{C}$ )}

Elle s'accompagne classiquement d'une absence de cellules germinales et de cellules de Sertoli, d'une atteinte de tous les composants de la paroi des tubes séminifères et des cellules de Leydig qui montrent un aspect caractéristique adénomateux, le nombre de ces cellules pouvant être augmenté ou plus souvent diminué. II n'est cependant pas rare que certains tubes séminifères contiennent des cellules de Sertoli et quelques cellules germinales, le plus souvent spermatogonies et spermatocytes I. Les formes les plus sévères de cette anomalie sont retrouvées dans le syndrome de Klinefelter, bien qu'une spermatogenèse puisse exister chez ces patients dans quelques tubes, avec production de spermatozoïdes.

\section{c) Les lésions diffuses de la spermatogenèse}

Elles forment un ensemble beaucoup plus hétérogène. Elles peuvent se manifester par une atteinte du compartiment ad luminal seul avec desquamation intraluminale de spermatides rondes (Figure 2A) ou, si l'atteinte est plus précoce, une desquamation intraluminale de spermatocytes I avec absence de spermatides (Figure 2B).

Plus fréquemment, s'associent des lésions du compartiment ad luminal et des lésions du compartiment basal. Deux cas de figure peuvent alors s'observer. Dans un premier cas, l'analyse histopathologique met en évidence une hypospermatogenèse pure avec diminution proportionnelle de tous les stades cellulaires
(Figure 2C), cette hypospermatogenèse peut aussi s'accompagner d'une desquamation des spermatocytes I avec modification des proportions relatives des différents types cellulaires. Cette anomalie est fréquemment observée dans les microdélétions AZFc. Dans un deuxième cas de figure, les lésions diffuses de la spermatogenèse se manifestent par un arrêt de maturation des spermatogonies, les spermatocytes sont peu nombreux et les spermatides rares (Figure 2D).

\section{d) L'atrophie mixte du testicule}

Elle se manifeste par la coexistence sur la même coupe, en proportions variables, de tubes $\mathrm{SCO}$, de tubes avec maturation des cellules germinales et de rares tubes hyalinisés.

\section{Les limites de l'histopathologie testiculaire}

a) Elles sont tout d'abord liées à la variabilité de l'interprétation d'un observateur à l'autre et aux variations qui peuvent exister au sein d'un testicule infertile. Ainsi des spermatozoïdes peuvent être recueillis dans $5 \%$ à $60 \%$ des cas de SCO. Par ailleurs, Coopeberg et al. [2] ont comparé les résultats de deux lecteurs indépendants de 132 biopsies testiculaires. Dans cette étude, il apparaît que les taux de concordance vont de $20 \%$ à $91 \%$ selon les pathologies, le taux le plus bas concernant les arrêts de maturation tardive de la spermatogenèse, le meilleur taux de concordance concernant les SCO.

b) Les limites de l'histopathologie testiculaire résultent aussi et surtout de l'impossibilité de poser un diagnostic étiologique sur l'aspect des lésions de la spermatogenèse, bien que certains aspects histopathologiques testiculaires soient préférentiellement associés une pathologie précise [10]. Des techniques complémentaires sont donc nécessaires pour apprécier les causes et les mécanismes des infertilités masculines de type non obstructif. Parmi ces techniques, I'hybridation in situ et l'immunocytochimie sur coupes histologiques permettent d'explorer les trois étapes de la spermatogenèse. L'étude de la méiose bénéficie des techniques cytogénétiques, moléculaires et immunocytochimiques, qui, couplées à l'identification des gènes de la méiose et de leur mutations permettent déjà d'identifier de nouvelles étiologies dans le groupe des infertilités masculines de type non obstructif. Malgré ces restrictions, l'histopathologie testiculaire est un complément indispensable des études méiotiques, elle doit être faite systématiquement lorsque le patient subit une biopsie testiculaire couplée à un acte de procréation médicalement assistée.

\section{ETUDE MEIOTIQUE}

La méiose est un événement fondamental au cours de 
la spermatogenèse dont la défaillance peut aboutir à un arrêt complet de la production des cellules germinales. Lors de la méiose se produisent deux événements étroitement liés l'un à l'autre qui conditionnent la survie des espèces et la variabilité des individus au sein d'une même espèce, ce sont : la recombinaison génétique et la réduction du nombre des chromosomes. Les grands événements de la méiose se déroulent lors de la première division, et plus particulièrement au cours de la prophase de la première division qui occupe à elle seule la presque totalité du temps imparti à la méiose. C'est au cours de cette étape de la méiose que se produiront les principales pathologies méiotiques, et c'est donc la prophase de la première division méiotique qui sera la scène sur laquelle apparaîtront les manifestations visibles des pathologies sous-jacentes. Les technologies modernes se sont surtout attachées à développer des moyens permettant d'identifier avec précision chacune des étapes de la prophase I afin de décrypter les mécanismes très complexes qui les soustendent et de mettre en évidence de nouvelles étiologies à l'infertilité masculine.

Nous avons développé dans notre laboratoire une technique d'étalement simple des cellules méiotiques à partir des prélèvements testiculaires réalisés dans le cadre de l'ICSI [8]. Après avoir prélevé les deux fragments testiculaires destinés à l'histologie, la totalité de la biopsie est envoyée au laboratoire de Fécondation in vitro, et c'est le matériel non utilisé pour l'ICSI, contenant les cellules germinales immatures, qui est récupéré pour la recherche. Ce matériel est conservé dans un milieu cryoprotecteur (HAM F10/10\% glycérol), dans l'azote liquide. L'utilisation d'une cytospin3 pour les étalements après décongélation des cellules nous permet de réduire au maximum la perte des cellules germinales, et de pouvoir ainsi étudier la méiose des patients présentant des hypospermatogenèses sévères.

\section{Rappel de la méiose $[4,5]$}

Notre rappel de la méiose se limitera à décrire le déroulement de la prophase de la première division. La méiose est précédée par une seule synthèse d'ADN caractérisée par sa longueur en comparaison de la synthèse d'ADN dans le cycle mitotique. Cette particularité est probablement et en partie liée au fait que les cassures double-brin qui initient la recombinaison génétique se produisent très vraisemblablement à la fin de la phase de synthèse d'ADN. Cette étape qui précède le démarrage de la méiose porte le nom de stade préleptotène. La prophase de la première division méiotique débute par l'individualisation des chromosomes, c'est le stade leptotène. Au cours du stade suivant ou stade zygotène, les chromosomes homologues s'apparient " gène à gène " sous forme de bivalents, en commençant par les extrémités, et lorsque l'appariement est achevé, la cellule est au stade pachytène. Au cours du stade diplotène, les chromosomes homologues se séparent excepté en des points précis appelés chiasmas et qui sont les témoins des crossing-overs. La prophase de la première division méiotique s'achève par la diacinèse, au cours de laquelle les bivalents se condensent et se détachent de la membrane nucléaire.

L'appariement des chromosomes au cours de la prophase I de la méiose résulte de la formation de structures protéiques sur lesquelles se fixent les homologues. Chacune de ces structures porte le nom de complexe synaptonémal (CS). II existe un CS pour chaque bivalent autosomique, qui s'étend sur toute la longueur du bivalent, et un petit complexe pour les chromosomes sexuels sur lequel s'apparient les régions pseudo-autosomales des chromosomes $X$ et $Y$. L'ultrastructure d'un CS et des homologues associés est schématisée Figure 3 . Le CS a la forme d'un rail comprenant un élément central et deux éléments latéraux sur lesquels sont fixés les chromosomes homologues. Les chromatides sœurs de chaque homologue sont maintenues cohésives par des protéines portant le nom de cohésines. Les éléments latéraux des complexes synaptonémaux apparaissent au stade leptotène, sous la forme d'éléments axiaux discontinus et mis en évidence par la technique d'immunocytochimie avec les mêmes anticorps que ceux utilisés pour visualiser les CS (Figure $4 \mathrm{~A}$ à $\mathrm{E}$ ).

La recombinaison génétique méiotique est un ensemble d'événements extrêmement complexes, qui débute par la formation des cassures double brin et s'achève par la résolution des crossing-over à la transition métaphase I - anaphase I. Dans un grand nombre d'espèces, dont l'homme, la recombinaison génétique et l'appariement des homologues sont deux événements étroitement intriqués, et les inactivations ciblées de gènes de l'un ou l'autre de ces événements se manifestent par les mêmes images méiotiques : asynapsis et fragmentation des bivalents. Actuellement, un grand nombre des événements de la recombinaison génétique peut être visualisé par des anticorps spécifiques de protéines impliquées dans ces événements. Nous avons développé le marquage des spots de recombinaison génétique par l'anticorps anti-MSH4, nous avons mis en évidence au stade leptotène et au pachytène précoce un grand nombre de spots correspondant aux sites d'appariement des homologues (Figure 5A), et au stade pachytène la localisation des crossing over (Figure $5 B)$. 

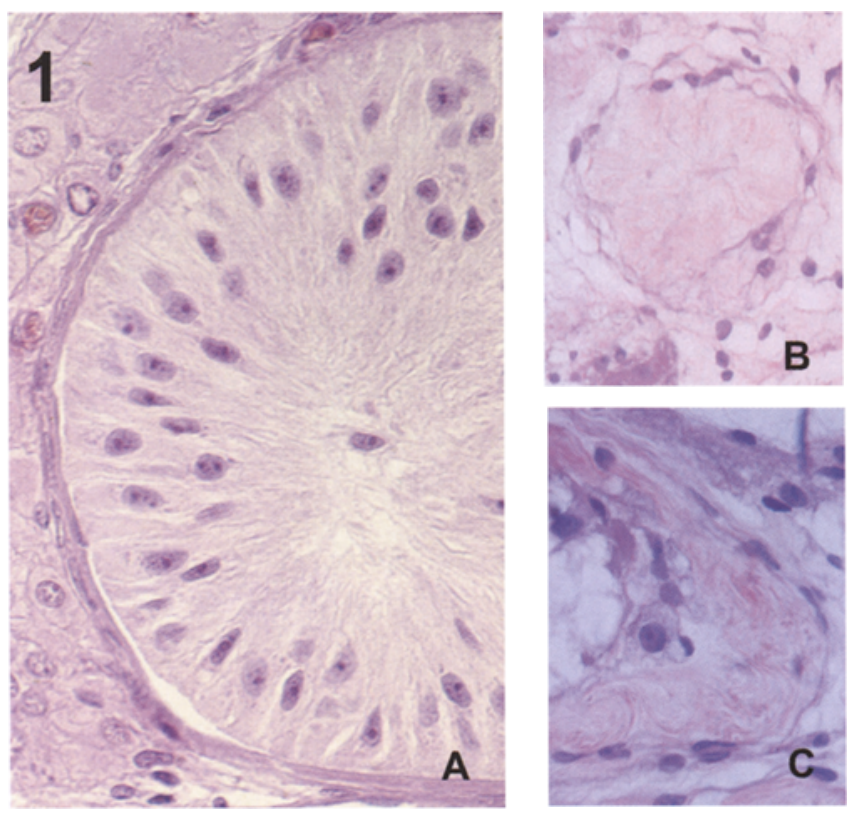

Figure 1 : histopathologie testiculaire. A - Sertoli Cell only. B - Hyalinisation tubulaire : absence lumière tubulaire, de cellules germinales et de cellules de Sertoli. C - Hyalinisation tubulaire : présence de rares spermatogonies et spermatocytes

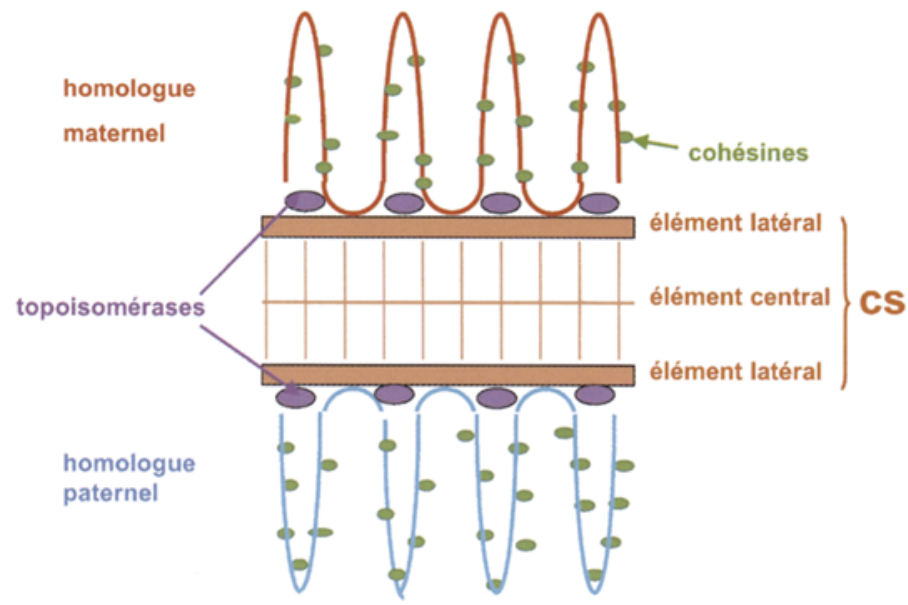

Figure 3 : Schéma d'un complexe synaptonémal (CS) sur lequel sont fixés les homologues maternels et paternels. Les chromatides scurs de ces homologues sont réunies par des protéines (cohésines) et fixées sur les éléments latéraux du CS par des protéines, parmi lesquelles les topoisomérases.
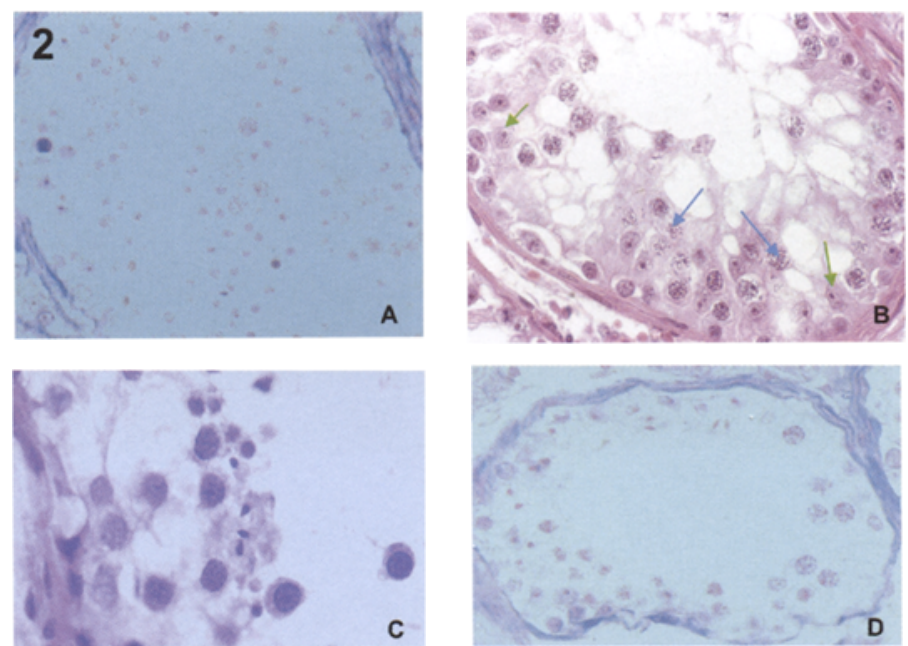

Figure 2 : histopathologie testiculaire. Lésions diffuses de la spermatogenèse. A - lésions du compartiment ad luminal avec desquamation intraluminale de spermatogonies rondes et de quelques spermatocytes. B - lésions du compartiment ad luminal avec desquamation intraluminale de spermatocytes I, absence de spermatides (flèches bleues = noyaux des spermatocytes I pachytènes; flèches vertes = noyaux des cellules de Sertoli). C - lésions des compartiments ad luminal et basal se manifestant par une hypospermatogenèse avec desquamation de spermatocytes I pachytènes. D - arrêt de maturation des spermatogonies, spermatocytes peu nombreux, rares spermatides.
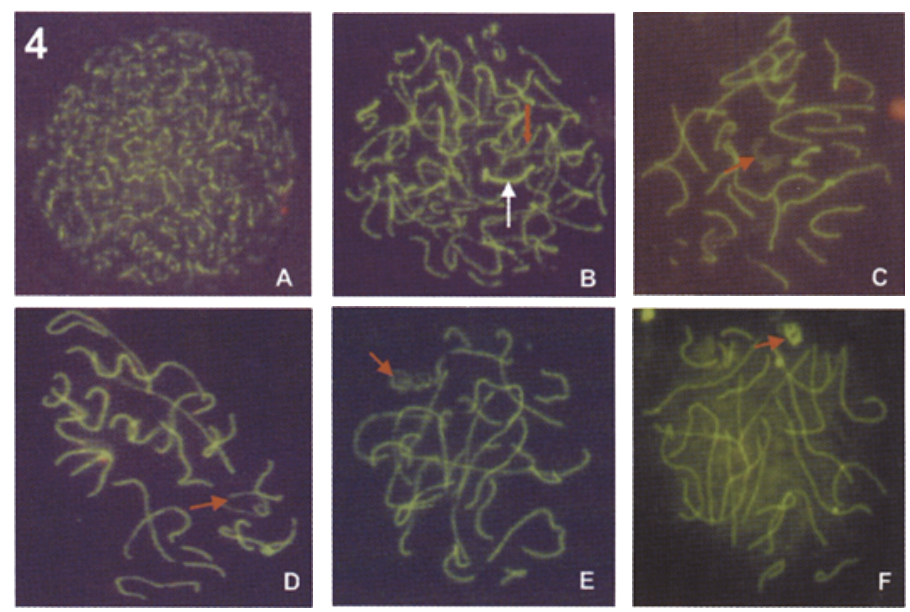

Figure 4 : les différents stades de la prophase I de la méiose normale, visualisés par l'anticorps SCP3.

A - stade leptotène : visualisation des éléments axiaux, discontinus. B - stade zygotène : début des appariements. On distingue nettement les éléments axiaux (flèche rouge) des complexes synaptonémaux (flèche blanche), résultant de l'appariement des éléments axiaux.

C - stade pachytène subdivisé en 4 sous-stades en fonction du degré croissant de compaction des chromosomes sexuels (flèches rouges) : $C=$ sous-stade $1, D=$ sousstade $2, E=$ sous-stade $3, F=V$ sous-stade 4. 

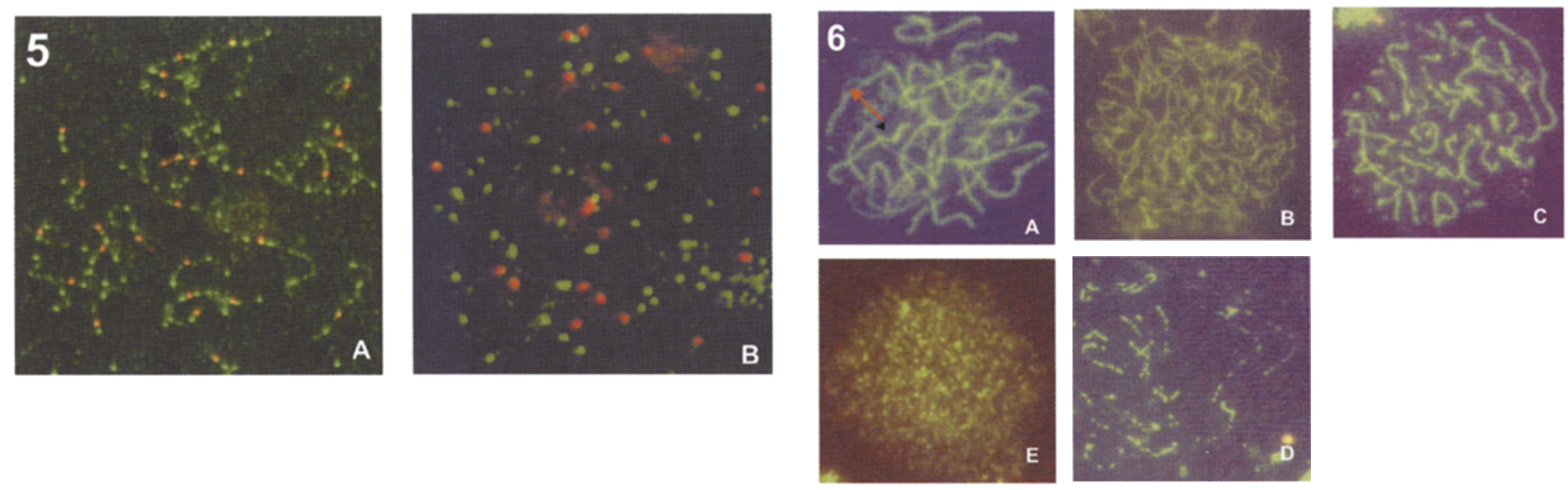

Figure 5 : A - visualisation des loci de recombinaison génétique par MSH4. B - visualisation de la localisation des crossing-over par le même anticorps (spots rouges = localisation des kinétochore ; spots verts = localisation de MSH4).

\section{Les anomalies de la prophase méiotique}

Notre démarche actuelle est d'analyser systématiquement toutes les biopsies testiculaires avec l'anticorps SCP3 quelque soit le résultat de l'histologie testiculaire. Le diagnostic histologique de syndrome SCO n'exclue pas la présence de cellules germinales et en particulier les stades qui nous intéressent plus particulièrement (du leptotène au pachytène). En effet, l'anticorps SCP3 révèle les spermatocytes $I$, du stade leptotène au stade diplotène. Au leptotène, SCP3 révèle les éléments axiaux sous forme de fins filaments discontinus (Figure 4A) ; au zygotène, les éléments axiaux sont continus et on voit apparaître les premiers complexes synaptonémaux (CS) (Figure 4B) ; au stade pachytène, on distingue nettement $22 \mathrm{CS}$ autosomiques et un bivalent sexuel dont la morphologie est différente en fonction du sous-stade pachytène.

En nous basant sur les travaux de Solari, nous distinguons 4 sous-stades : $X Y 1$, dans lequel il existe un petit $C S$ au niveau des régions pseudo-autosomales des chromosomes $X$ et $Y$ (Figure 4C), les éléments axiaux des régions différentielles étant non appariées et non condensées ; $X Y 2$, dans lequel les axes des chromosomes $X$ et $Y$ commencent à s'enrouler sur euXmêmes (Figure 4D) ; XY3, dans lequel le degré d'enroulement des axes est important (Figure 4E) et $X Y 4$ dans lequel les chromosomes $X$ et $Y$ sont complètement compactés (Figure 4F). Chez les sujets à spermatogenèse normale, les spermatocytes aux stades leptotène, zygotène et diplotène sont rares sur les étalements, alors que les spermatocytes au stade

Figure 6 : Anomalies méiotiques.

A-asynapsis partiel (flèche rouge).

$B$ - asynapsis total.

$C-$ Bivalents fragmentés.

$D$ - bivalents en pointillés.

E-Pulvérisation de complexes synaptonémaux.

pachytène sont extrêmement fréquents. Parmi eux, les sous-stades tardifs, $X Y 3$ et $X Y 4$, sont plus fréquemment représentés que les sous-stades précoces, $X Y 1$ et $X Y 2$ : $53,9 \%$ pour la somme des $X Y 3$ et XY4 vs $46 \%$ pour la somme des $X Y 1$ et $X Y 2$ dans notre étude [6].

Avec l'anticorps SCP3, nous décrivons actuellement 3 types d'anomalies : les anomalies de l'appariement des homologues ou asynapsis (Figure $6 \mathrm{~A}$ et $\mathrm{B}$ ), la fragmentation des CS (Figure $6 \mathrm{C}$ et $\mathrm{D}$ ) et les anomalies de condensation des CS (Figure 6E). Nous avons analysé la méiose chez 41 patients infertiles qui ont eu une biopsie testiculaire, soit pour bilan de leur infertilité, soit dans le cadre d'une ICSI, et chez 13 sujets contrôles [6]. Parmi les patients, 19 avaient une infertilité de type obstructif $(O)$ et 22 patients avaient une infertilité de type non obstructif (NO). Nous avons évalué le pourcentage de noyaux présentant des asynapsis pour chacun de ces patients. Nous avons montré que cette anomalie de la méiose existait chez les contrôles comme chez les patients, mais que la fréquence de cette anomalie variait en fonction des groupes. En effet, le pourcentage d'asynapsis dans le groupe témoin était de $10,95 \%$, et de $7,9 \%$ dans le groupe $O$, la différence entre ces deux groupes n'était pas significative. Le pourcentage d'asynapsis dans le groupe NO était de $22,3 \%$, et la différence avec les deux autres groupes était très hautement significative. Deux patients du groupe NO montraient des taux d'asynapsis très étendus et très hautement supérieurs à ceux des autres patients de ce groupe (respectivement $86 \%$ et $91,8 \%$ ). Nous avons conclu que ces patients présentaient 
probablement une cause génétique à leur infertilité, nous avons en effet mis récemment en évidence une microdélétion AZFC chez l'un de ces patients.

A partir de cette étude, nous avons évalué la fréquence des anomalies primitives de la méiose à $9 \%$ des infertilités de type NO. Les taux moyens observés chez les autres patients du groupe NO résultent probablement d'une souffrance des cellules germinales secondaire à une altération de leur environnement testiculaire. Nous avons ensuite évalué les pourcentages respectifs des différents sous-groupes du stade pachytène dans les deux groupes de patients, et nous avons montré que, contrairement aux sujets témoins, il y avait une très nette prédominance des sous-stades précoces (XY1 et $X Y 2$ ) par rapport aux sous-stades tardifs (XY3 et $X Y 4)$, quel que soit le groupe de patients et quelque soit l'étiologie de l'infertilité dans chaque groupe. Nous en avons conclu que le point de contrôle du stade pachytène était situé au milieu de ce stade.

Nous avons ensuite étudié le comportement des chromosomes méiotiques d'un patient présentant une microdélétion AZFb [9] avec l'anticorps SCP3. Nous avons mis en évidence une accumulation de stades leptotène ( $21 \%$ des noyaux analysés), un fort taux de noyaux pachytène présentant des asynapsis étendus voire totaux $(69 \%)$, et un blocage de la méiose au stade pachytène moyen ; le pourcentage de sous stades $X Y 1$ et $X Y 2$ était de $98,5 \%$, seuls 2 noyaux étaient observés au sous-stade $X Y 3(1,5 \%)$. Il était jusqu'à présent classiquement admis que les asynapsis étaient responsables de l'arrêt de la spermatogenèse pour les cellules qui présentaient ce type d'anomalie. La présence de $29 \%$ de noyaux sans asynapsis nous a fait conclure que chez ce patient, les asynapsis n'étaient pas responsables de l'arrêt méiotique. II est donc plus probable que l'arrêt méiotique et les asynapsis résultent de tous deux d'une cause commune liée à la présence de la microdélétion AZFb.

Nous avons pensé que ces asynapsis pouvaient traduire l'existence d'une défaillance de la recombinaison génétique. En effet, les expériences d'inactivations ciblées de gènes de la recombinaison génétique réalisés chez la levure se manifestent par la présence d'asynapsis. Nous avons réalisé un immunomarquage avec l'anticorps $\mathrm{H} 2 \mathrm{AX}$ qui visualise la forme phosphorylée de la protéine $\mathrm{H} 2 \mathrm{AX}, \mathrm{gH} 2 \mathrm{AX}$. La présence de la forme phosphorylée de cette protéine est le témoin de la réparation des cassures double-brin qui initient la recombinaison génétique. Nous avons obtenu un marquage normal avec cet anticorps, nous pouvons donc conclure que la première étape de la recombinaison génétique se déroule normalement chez ce patient. II ne s'agit là que d'un cas, mais d'autres études méiotiques chez les patients présentant une microdélétion AZFb sont en cours afin d'étendre ces conclusions à toutes les microdélétions $\mathrm{AZFb}$.

\section{CONCLUSION}

Lorsqu'une biopsie testiculaire est réalisée dans le cadre d'une ICSI, il apparaît maintenant indispensable de prélever systématiquement un fragment de pulpe testiculaire pour réaliser une étude histopathologique testiculaire. II est absolument nécessaire que l'analyse soit faite par un biologiste averti qui prenne le temps d'analyser avec rigueur un nombre de tubes suffisants pour ne pas prendre le risque de laisser passer des zones très limitées de spermatogenèse, et de faire une étude quantitative des cellules germinales. Cette analyse peut être faite par tous les centres de reproduction qui peuvent toujours disposer d'un laboratoire d'anatomie pathologique à proximité. II n'en n'est pas de même pour l'étude méiotique pour laquelle il existe peu de biologistes formés à son étude et qui n'est pas un examen codifié. Cette étude est donc actuellement réalisée dans le cadre de la recherche, elle a l'avantáge de ne faire courir aucun risque au patient puisqu'elle est réalisée sur du matériel destiné à être jeté. C'est une analyse qui apporte des renseignements irremplaçables sur le comportement des chromosomes méiotiques et sur un certain nombre de protéines qui agissent à ce niveau. Elle fera très certainement progresser l'identification des mécanismes de l'infertilité masculine mais il n'est pas possible de dire actuellement quelle place elle pourra occuper dans les laboratoires de biologie de la reproduction, et si cet examen sera dans le futur systématiquement demandé dans un but diagnostique, devant une infertilité d'étiologie inconnue.

Remerciements:

Nous remercions Peter Moens, Barabara Spyropoulos et Christa Heyting pour nous avoir donné l'anticorps COR1/SCP3. Nous remercions Véronique Paquis qui nous a donné l'anticorps MSH4. Nous remercions aussi les techniciennes de l'UF 3201, qui participent à ces travaux: Danièle Daioglou, Catherine Metton et Marie-Josée FaysBernardin.

\section{REFERENCES}

1. CLERMONT Y. : Kinetic of spermatogenesis in mammals : seminiferous epithelium cycle and spermatogonial renewal. Physiol. Review, 1972, 1 : 198-235.

2. COOPERBERG M.R., CHIT JAD A., CHA I., TUREK P.J. : 
Variability in testis biopsy interpratation : implications for male infertility care in the era of intracytoplasmic sperm injection. Fertil. Steril., 2005, $84: 672-677$.

3. DAKOUANE M., BICCHIERAY L., BERGERE M., ALBERT M., VIALARD F., SELVA J. : A histomorphometric and cytogenetic study of testis from men 29-102 old. Fertil. Steril., 2005, 83 : 923-928.

4. GUICHAOUA M.R., LUCIANI J.M. : Méiose. In : Feingold J., Fellous M., Solignac M. Principes de Génétique Humaine. Hermann Editeur des Sciences et des Arts, 1998 : 59-68.

5. GUICHAOUAM.R. La méiose spermatocytaire et ses anomalies. In : Blanc B., Porcu G. eds. Stérilité. Editions Arnette, 2002 : 355-368.

6. GUICHAOUA M.R., PERRIN J., METZLER-GUILLEMAIN C., SAIAS-MAGNAN J., GIORGI R., GRILLO J.M. : Meiotic anomalies in infertile men with a severe defect in spermatogenesis. Hum. Reprod., 2005, 20 : 1897-1902.

7. LA VALETTE, St. G. von. Ueber die genese der samenkorper 4. Arch. Mikroskop. Anat., 1876, 12 : 797-825.

8. METZLER-GUILLEMAIN C., GUICHAOUA MR. : A simple and reliable method for meiotic studies on testicular samples used for intracytoplasmic sperm injection. Fertil. Steril., 2000, 74 : 916-919.

9. PERRIN J., METZLER-GUILLEMAIN C., KARSENTY G., GRILLO J.M., MITCHELL M.J., GUICHAOUA M.R. : Meiotic arrest at mid pachytene stage in a patient bearing complete AZFb deletion of the Y chromosome. Fertil. Steril., 2006, 85 : 494.e5 - 494.e8.

10. NISTAL M., PANIAGAR. : Testicular biopsy. Uropath., 1999, 26 : 555-593.

Présentation au XXIIème congrès de la SALF Marseille 2005

Manuscrit reçu : février 2006 ; accepté avril 2006.

\section{ABSTRACT}

Testicular histology and meiotic studies in nonobstructive sterility

\section{Rahma GHALAMOUN-SLAIMI, Marie-Roberte GUICHAOUA}

There has been a renewed interest in testicular biopsy to evaluate infertility since the introduction, in 1993, of ICSI in azoospermic men with testicular sperm extraction (TESE) and intracytoplasmic sperm injection for the treatment of obstructive azoospermia. TESE is now performed for the treatment of nonobstructive azoospermia, and the testicular material sampled for therapeutic purposes can also be used for diagnostic and research purposes. The development of new methods of investigation of spermatogenesis, such as immunocytochemistry and fluorescent in situ hybridization (FISH) have also led to a renewed interest in analysis of spermatogenesis on testicular biopsy. A precise "testicular phenotype" must now be established to propose an aetiological diagnosis, and to determine the mechanisms and risks of nonobstructive azoospermia and severe oligozoospermia for the embryo. We systematically perform testicular histopathology and meiotic study for each patient undergoing testicular biopsy for ICSI.

We first describe the histopathological lesions. Examination of the testicular biopsy specimen determines whether the lesion is focal or diffuse. If it is focal, the percentage of altered tubules, evaluated on $\mathbf{5 0}$ tubules, should be calculated. Quantitative evaluation of seminiferous epithelium and a qualitative study of cell morphology must also be performed. There are four frequent lesion patterns: 1- Sertoli-cell-only syndrome; 2- tubular hyalinisation; 3- diffuse lesions in spermatogenesis; 4- mixed atrophy. However, the reliability of interpretation of testicular histology presents certain limitations, as no standard method of analysis of testicular biopsies has been defined and there is a marked variability in the histologist's capacity to recognize the various histological patterns.

Meiotic study is performed on the cell suspension remaining after ICSI, which contains immature germ cells. New methods using immunocytochemistry have replaced older methods. The panel of antibodies which detect individual protein components at different stages of meiosis provides a valuable tool for the detection and interpretation of abnormal meiotic profiles. We performed meiotic studies on 41 patients and 13 controls after Giemsa staining, and synaptonemal complexes (SC) from nine of these patients and one control were immunostained with a polyclonal antibody which recognizes the COR1/SCP3 protein of the lateral element of the SC. Nineteen of the patients presented obstructive infertility $(O)$ and 22 presented nonobstructive infertility (NO). We showed that the rate of asynaptic nuclei from the NO group $(25.4 \%)$ was significantly higher than that of the $O$ group $(9.8 \%)$ and the controls $(9.8 \%)$. Two patients of the NO group had a high percentage of asynaptic nuclei ( $86 \%$ and $91.8 \%$ ), which could arise from a primary meiotic defect. One of these patients had an AZFc microdeletion. The meiotic study in a patient with classical complete AZFb microdeletion revealed a high prevalence of early meiotic stages: leptotene, zygotene and early pachytene stages and marked impairment of the synaptic process in most spermatocytes. In the light of these findings, we conclude that the pachytene checkpoint is localized at the mid-pachytene stage in humans.

Key words: azoospermia, oligozoospermia, testicular histology, meiosis, asynapsis 